\title{
Malaria Parasite Density Estimated with White Blood Cells Count Reference Value Agrees with Density Estimated with Absolute in Children Less Than 5 Years in Central Ghana
}

\author{
Dennis Adu-Gyasi, Kwaku Poku Asante, Sam Newton, Sabastina Amoako, David Dosoo, \\ Love Ankrah, George Adjei, Seeba Amenga-Etego, and Seth Owusu-Agyei
}

Kintampo Health Research Centre (KHRC), Box 200, Kintampo North, Brong Ahafo, Ghana

Correspondence should be addressed to Dennis Adu-Gyasi; adugyasidennis@yahoo.co.uk

Received 5 January 2015; Accepted 9 March 2015

Academic Editor: Polrat Wilairatana

Copyright (C) 2015 Dennis Adu-Gyasi et al. This is an open access article distributed under the Creative Commons Attribution License, which permits unrestricted use, distribution, and reproduction in any medium, provided the original work is properly cited.

\begin{abstract}
Introduction. The estimation of malaria parasite density using a microscope heavily relies on White Blood Cells (WBCs) counts. An assumed WBCs count of $8000 / \mu \mathrm{L}$ has been accepted as reasonably accurate in estimating malaria parasite densities due to the challenge to accurately determine WBCs count. Method. The study used 4944 pieces of laboratory data of consented participants of age group less than 5 years. The study compared parasite densities of absolute WBCs, assumed WBCs, and the WBCs reference values in Central Ghana. Ethical approvals were given by three ethics committees. Results. The mean $( \pm \mathrm{SD})$ WBCs and geometric mean parasite density (GMPD) were $10500 / \mu \mathrm{L}( \pm 4.1)$ and $10644 / \mu \mathrm{L}(95 \% \mathrm{CI} 9986 / \mu \mathrm{L}$ to $11346 / \mu \mathrm{L})$, respectively. The difference in the GMPD compared using absolute WBCs and densities of assumed WBCs was significantly lower. The difference in GMPD obtained with an assumed WBCs count and that of the WBCs reference values for the study area, $10400 / \mu \mathrm{L}$ and $9200 / \mu \mathrm{L}$ for children in different age groups, were not significant. Discussion. Significant errors could result when assumed WBCs count is used to estimate malaria parasite density in children. GMPD generated with WBCs reference values statistically agreed with density from the absolute WBCs. When obtaining absolute WBC is not possible, the reference value can be used to estimate parasite density.
\end{abstract}

\section{Background}

Malaria is caused by one or a combination of four species of Plasmodia and leads to over one million deaths, of which over $75 \%$ occur in African children under 5 years infected mainly with Plasmodium falciparum (Pf). Pf and Plasmodium vivax $(P v)$ are identified as the Plasmodium species responsible for causing the most severe form of malaria [1].

In epidemiological studies, intervention studies, and clinical trials, malaria microscopy is routinely relied upon as a primary endpoint measurement of the level of malaria infection [2]. This is expressed as parasite density and is classically defined as the number of asexual forms of parasite relative to a blood volume (e.g., microliter) [3]. In the four basic counting techniques using microscopy [3], White Blood Cells (WBCs) are relatively used in estimating Plasmodium parasitaemia by counting the number of parasites against a predetermined number of WBCs on Giemsa stained blood smears.

Complete blood counts, particularly WBCs count, can be performed with new generation automated haematology analysers [4] and/or manually using stained microscope smears and the Neubauer chamber and counters $[5,6]$. Some health facilities frequently use manual convention methods to determine the complete blood counts of a patient for management as a result of a high cost of purchasing and maintaining fully automated or semiautomated haematology analyzers. Another cost burden using an automated system includes the ability to ensure prompt validation, maintenance, and implementation of rigorous quality systems. Automated machines may therefore not be the preferred choice to quantify WBCs in resource poor areas.

Due to the frequent lack of facilities in some malaria endemic countries to quantify WBCs, an assumed WBCs 
count of $8000 / \mu \mathrm{L}$ of blood has been accepted by World Health Organization as reasonably accurate [7] to estimate malaria parasite densities. Assumed WBCs count of blood may generate systematic errors which could produce incorrect conclusions in patient management or during clinical research that uses malaria parasite counts as an end point $[3,8]$.

Medical laboratories need to set relevant reference ranges for WBCs and other common laboratory parameters for clinical management of patients or during malaria clinical research [9]. Kintampo Health Research Centre has established reference values for common haematological and biochemical laboratory parameters for its study area covering an area of about $7200 \mathrm{~km}^{2}$ [10].

The team compared the geometric mean parasite densities (GMPD) calculated using absolute WBCs, assumed WBCs, and WBCs reference values of the participants enrolled in the malaria studies in middle Ghana to assess the impact of using these as malaria parasite density estimators.

\section{Methods}

2.1. Ethics Statement. The studies used for this data analysis were approved by the ethical review committees of the Ghana Health Service, London School of Hygiene and Tropical Medicine, Kintampo Health Research Centre Institutional Ethics Committee, Noguchi Memorial Institute of Medical Research, and The Ghana Food and Drugs Authority. Written informed consent was sought from all mothers whose children participated in the studies.

2.2. Site Description. The data analysis was carried out on laboratory data from a study area with a profile presented by Adu-Gyasi et al., 2012 [8]. Briefly, the area is located within the forest-savannah transitional ecological zone in Ghana with perennial malaria transmission [11]. $P f$ is the predominating Plasmodium species while P. malariae and $P$. ovale are in the minority.

2.3. Description of Data Sources. The data was obtained from children under 5 years who were recruited into malarial studies carried out by Kintampo Health Research Centre between the periods of October 2008 and March 2011. In the first study, children were enrolled and followed actively every month for two years. The children were referred to the hospital for care whenever they were unwell to determine the incidence of malaria [12]. The second study which was a clinical trial was to determine the effect of providing Micronutrient Powder with or without iron on the incidence of malaria among children under three years living in a high malaria-burden area in Ghana [13].

2.4. Blood Sample Collection and Processing. In each of the studies, blood samples were collected by finger-prick into $0.5 \mathrm{~mL}$ microtainers containing ethylenediaminetetraacetic acid (K2EDTA-BD, USA) from the participants on their scheduled visit days and on any other day participants visited the health facilities with illness. Samples were collected and transported according to the Standard Operating Procedures
(SOPs) established by the Kintampo Health Research Centre. Briefly, participant's finger was rubbed, and the tip was cleaned with disposable alcohol swab and allowed to dry. Safety lancet was used to prick and drops of blood were collected into labelled test-tubes to the required volume. Collected samples were used for full blood count analysis and for making blood smears. Examination of thick and thin smears was carried out as described by Adu-Gyasi et al., 2012 [8].

2.5. Estimation of Parasite Density. Parasite densities for all participants were calculated using assumed WBCs count of $5000 / \mu \mathrm{L}, 6000 / \mu \mathrm{L}, 8000 / \mu \mathrm{L}$, and $10000 / \mu \mathrm{L}$ of blood. In addition, we used the WBCs reference values established for children aged less than one year $(10000 / \mu \mathrm{L}$ of blood) and also for children less than five years $(9200 / \mu \mathrm{L})[14]$.

2.6. Data Entry, Cleaning, and Analyses. Data obtained from the database of the malarial studies was checked for completeness and consistency and all queries were resolved. All parasite negative blood slide results were removed before analysis. Analyses were done using STATA (version 12; Stata Corp., TX, USA) and GraphPad PRISM version 5.0 (GraphPad Software, Inc.) particularly for geometric mean and intervals. Geometric means at 95\% CI that did not overlap were considered significant.

\section{Results}

Of the 39851 results received over the study period, data of 4944 participant results were consistent and complete for the purpose of our analysis. Of the total, 50.6\% (2498/4944) were males and $49.4 \%(2443 / 4944)$ were females.

The mean $( \pm$ SD) WBCs and GMPD of the 4944 positive samples were $10500 / \mu \mathrm{L}( \pm 4.1)$ and $10644 / \mu \mathrm{L}(95 \%$ CI $9986 / \mu \mathrm{L}$ to $11347 / \mu \mathrm{L}$ ) of blood, respectively. There was no significant difference between the mean (SD) WBCs $10600 / \mu \mathrm{L}$ (4.0) for males and $10500 / \mu \mathrm{L}( \pm 4.1)$ for females $(P=0.255)$. Neither was there a significant difference between the GMPD $11058 / \mu \mathrm{L}(95 \%$ CI $10114 / \mu \mathrm{L}$ to $12091 / \mu \mathrm{L}$ ) of blood for males (Table 1) and $10217 / \mu \mathrm{L}(95 \% \mathrm{CI} 9324 / \mu \mathrm{L}$ to $11197 / \mu \mathrm{L})$ of blood for females (Table 2).

With an assumed WBCs count of $8000 / \mu \mathrm{L}$, a GMPD of $8679 / \mu \mathrm{L}(95 \% \mathrm{CI}, 8140 / \mu \mathrm{L}$ to $9253 / \mu \mathrm{L})$ of blood was estimated (Table 3).

In using the WBCs reference values established among children in the study area, $(10400 / \mu \mathrm{L})$, a GMPD of $11282 / \mu \mathrm{L}$ ( $95 \%$ CI, $10581 / \mu \mathrm{L}$ to $12029 / \mu \mathrm{L}$ ) of blood and a WBCs count of $9200 / \mu \mathrm{L}$ produced a GMPD of $9981 / \mu \mathrm{L}(95 \% \mathrm{CI}, 9361 / \mu \mathrm{L}$ to $10641 / \mu \mathrm{L}$ ) of blood.

The difference in the GMPD calculated using absolute WBCs compared to densities estimated with assumed WBCs was significantly lower for $8000 / \mu \mathrm{L}$. However, GMPD for assumed WBCs count of $10.0 \times 10^{9} / \mathrm{L}$, WBCs reference values of $10400 / \mu \mathrm{L}$, and $9200 / \mu \mathrm{L}$ estimated a geometric mean parasite density of $10848 / \mu \mathrm{L}$ (95\% CI, $10175 / \mu \mathrm{L}$ to $11567 / \mu \mathrm{L})$, $11282 / \mu \mathrm{L}(95 \% \mathrm{CI}, 10581 / \mu \mathrm{L}$ to $12029 / \mu \mathrm{L})$, and $9981 / \mu \mathrm{L}(95 \%$ CI, $9361 / \mu \mathrm{L}$ to $10641 / \mu \mathrm{L}$ ) of blood that was not significantly different from estimates obtained with the absolute WBCs 


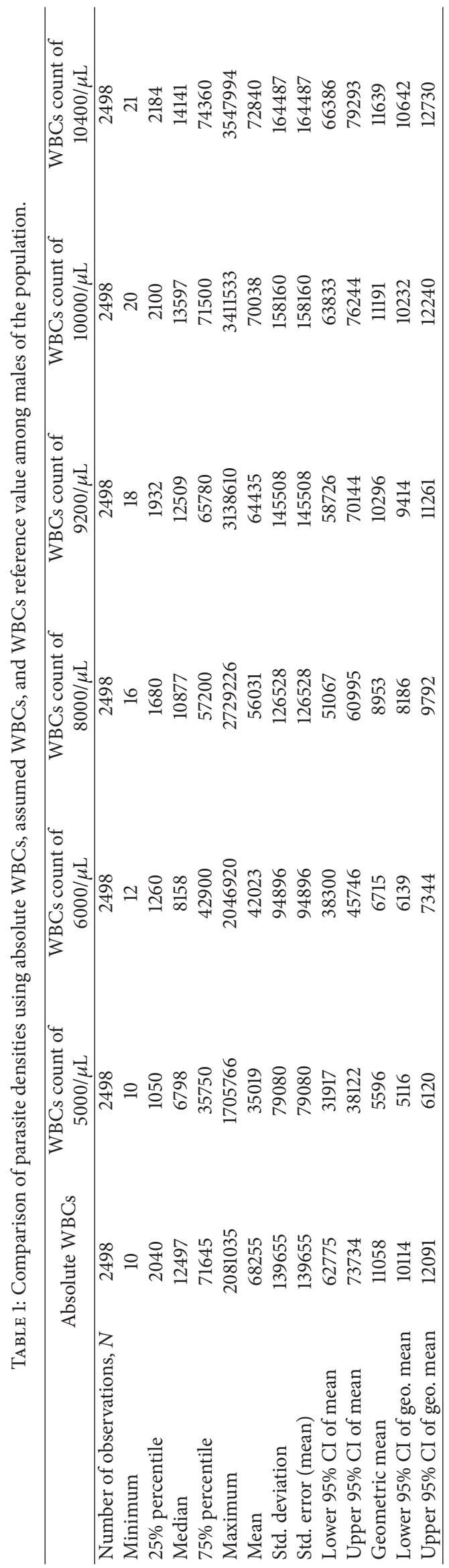




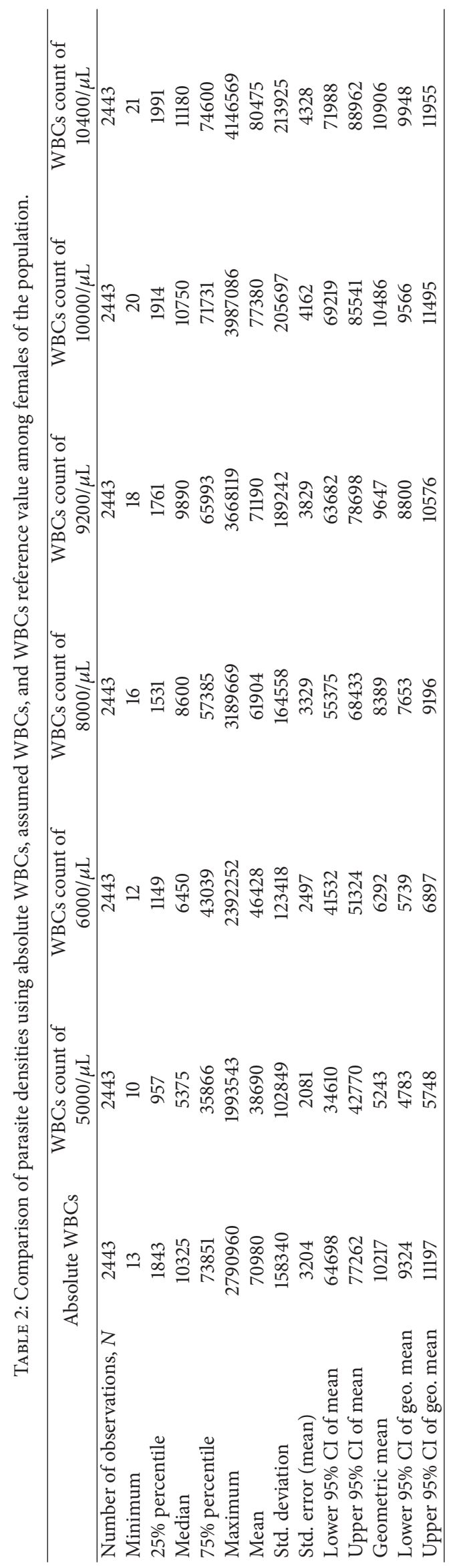




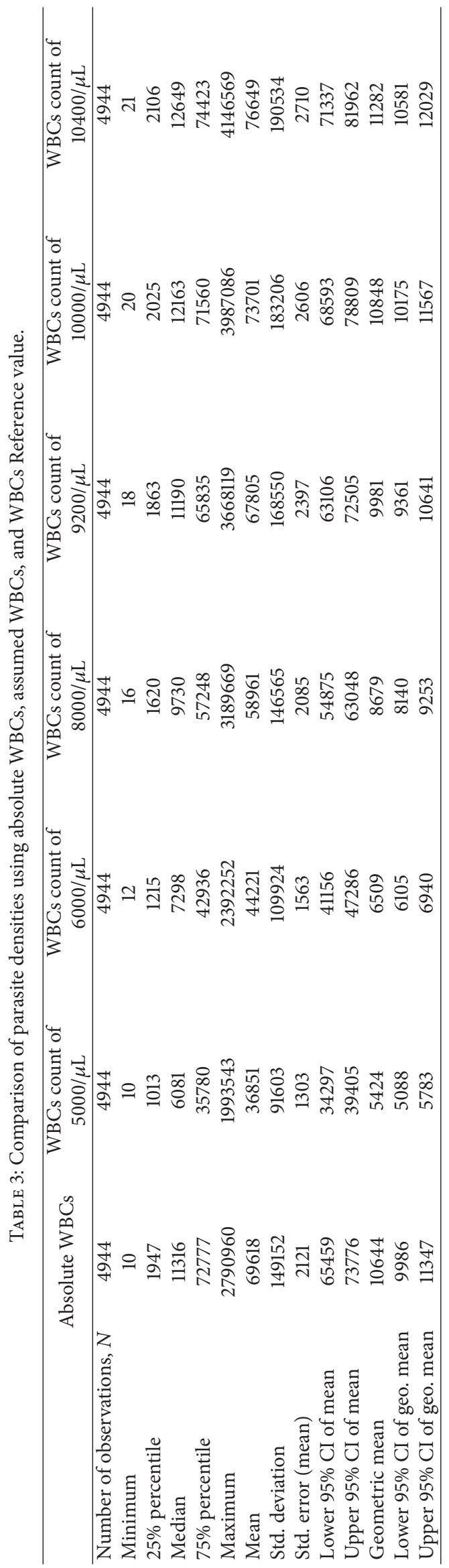




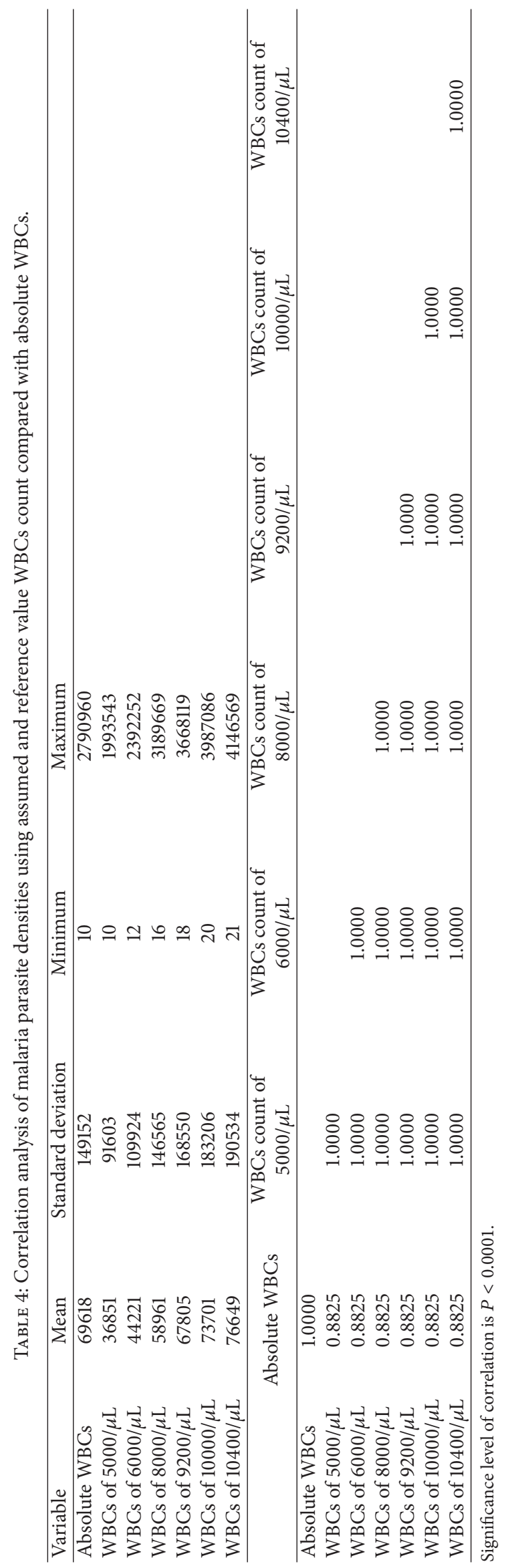


(Table 3). The interval of GMPD obtained when an assumed WBCs of $8000 / \mu \mathrm{L}, 6000 / \mu \mathrm{L}$ and $5000 / \mu \mathrm{L}$ were used and that from absolute WBCs count did not overlap. However, the intervals of GMPD from absolute WBCs count and that obtained with assumed WBCs counts of $9200 / \mu \mathrm{L}, 10000 / \mu \mathrm{L}$ and $10400 / \mu \mathrm{L}$ overlapped showing no significant difference in the GMPD (Table 3).

A correlation analysis of the malaria parasite densities with the WBCs reference value and assumed WBCs compared to the absolute WBCs showed a significant level of correlation $(P<0.0001)$ with all the assumed WBCs count (Table 4).

\section{Discussion}

Malaria parasite density is necessary for patient management. This has become dominated by the convenient but inaccurate assumption of a constant WBCs count of $8000 / \mu \mathrm{L}$ of peripheral blood $[3,15]$ due to lack of capacity to measure patients absolute WBCs [16].

The mean WBCs count from the participants with malaria infection compares with the WBCs reference values, $10400 / \mu \mathrm{L}$ for children less than 1 year and $9200 / \mu \mathrm{L}$ for children up to 5 years [14]. The parasite densities from the WBCs reference values agree with the density obtained from using participant's absolute WBCs.

It is agreed that the best solution in estimating parasite densities would be to use the corresponding absolute WBCs count for each age group [17]. Though there is a significant level of correlation, estimating the parasite density of Plasmodium species with the assumed WBCs count of $8000 / \mu \mathrm{L}$ of blood [7], compared to the absolute and reference value WBCs count, would mean underestimating significantly the parasite density of Plasmodium species infections for patients in the study area. Therefore, establishing regional based reference WBCs as suggested [17] to estimate parasite densities in malarial infections will be appropriate. This was evident in the fact that the geometric mean parasite density, $11282 / \mu \mathrm{L}(95 \% \mathrm{CI}, 10581 / \mu \mathrm{L}$ to $12029 / \mu \mathrm{L})$ and $9981 / \mu \mathrm{L}(95 \%$ $\mathrm{CI}, 9361 / \mu \mathrm{L}$ to $10641 / \mu \mathrm{L})$ of blood, estimated by using the established WBCs standard values of $10,400 / \mu \mathrm{L}$ and $9200 / \mu \mathrm{L}$ of blood for children less than 1 year and up to 5 years old, respectively, overlaps with the geometric mean parasite density obtained by using the mean absolute WBCs count, $10644 / \mu \mathrm{L}(95 \%$ CI $9986 / \mu \mathrm{L}$ to $11347 / \mu \mathrm{L})$ of blood, of the participants. Results obtained when an assumed WBCs count of $10000 / \mu \mathrm{L}$ of blood was used to estimate the parasite density [8] were also consistent with that from the absolute WBCs count of participants. The parasitaemia obtained by the use of the other assumed WBCs count was significantly lower (Table 3) [8].

\section{Conclusions}

Since lack of resources in some settings in Africa makes it difficult to estimate malaria parasite density based on actual WBCs count of patients, where available, the study recommends the use of an established WBCs reference value for a known population and where the reference value could be implied. The reference value established in Kintampo has been predicted as a malaria density estimator when compared with the absolute WBCs of participants. In environments where reference values have not been established, the study affirms the use of an assumed WBCs count of $10000 / \mu \mathrm{L}$ of blood to estimate malaria parasite density as documented [8].

\section{Limitations of the Study}

The ages of all participants were not stratified into groups. This did not make it possible to compare differences at the possible age groups less than 5 years.

\section{Conflict of Interests}

The authors declare that they have no competing interests.

\section{Authors' Contribution}

Dennis Adu-Gyasi, Kwaku Poku Asante, Sabastina Amoako, David Dosoo, and Love Ankrah designed the data analysis plan, performed most of the experiments, and cowrote the paper. George Adjei and Seeba Amenga-Etego contributed to the study design, performance of experiments, and statistical analysis and helped in writing the paper. Sam Newton and Seth Owusu-Agyei designed the experiments, supervised the study, and cowrote the paper. All authors read and approved the final version of the paper.

\section{Acknowledgments}

The study team wish to thank the chiefs, opinion leaders, and elders of participating communities, Wenchi Municipal, and Tain District Health Management Team, especially those who participated in the malarial based studies in Kintampo Health Research Centre (KHRC). The authors' appreciation also goes to the staff of Kintampo Health Research Centre who assisted in this work. Kintampo Health Research Centre is a member site of the INDEPTH Network. The study team thank Grace W. Mwangoka, Kwadwo Boateng, and Ms. Portia Amoah for their motivations towards the development of this paper.

\section{References}

[1] E. Tjitra, N. M. Anstey, P. Sugiarto et al., "Multidrug-resistant Plasmodium vivax associated with severe and fatal malaria: a prospective study in Papua, Indonesia," PLoS Medicine, vol. 5, no. $6, \mathrm{e} 128,2008$.

[2] W. P. O’Meara, M. Barcus, C. Wongsrichanalai et al., "Reader technique as a source of variability in determining malaria parasite density by microscopy," Malaria Journal, vol. 5, article $118,2006$.

[3] I. Hammami, G. Nuel, and A. Garcia, "Statistical properties of parasite density estimators in Malaria," PLoS ONE, vol. 8, no. 3, Article ID e51987, 2013.

[4] J. van den Bossche, K. Devreese, R. Malfait et al., "Reference intervals for a complete blood count determined on different automated haematology analysers: Abx Pentra 120 Retic, Coulter Gen-S, Sysmex SE 9500, Abbott Cell Dyn 4000 and Bayer Advia 120," Clinical Chemistry and Laboratory Medicine, vol. 40, no. 1, pp. 69-73, 2002. 
[5] C. Briggs, P. Harrison, and S. J. Machin, "Continuing developments with the automated platelet count," International Journal of Laboratory Hematology, vol. 29, no. 2, pp. 77-91, 2007.

[6] E. Petersen, N. T. Marbiah, L. New, and A. Gottschau, "Comparison of two methods for enumerating malaria parasites in thick blood films," American Journal of Tropical Medicine and Hygiene, vol. 55, no. 5, pp. 485-489, 1996.

[7] WHO, Basic Malaria Microscopy, World Health Organization, Geneva, Switzerland, 2010, http://whqlibdoc.who.int/publications/2010/9789241547826_eng.pdf.

[8] D. Adu-Gyasi, M. Adams, S. Amoako et al., "Estimating malaria parasite density: assumed white blood cell count of $10,000 / \mu \mathrm{l}$ of blood is appropriate measure in Central Ghana," Malaria Journal, vol. 11, article 238, 2012.

[9] K. Koram, M. Addae, J. Ocran, S. Adu-amankwah, W. Rogers, and F. Nkrumah, "Population based reference intervals for common blood haematological and biochemical parameters in the Akuapem north district," Ghana Medical Journal, vol. 41, no. 4, 2010 .

[10] D. K. Dosoo, K. Kayan, D. Adu-Gyasi et al., "Haematological and biochemical reference values for healthy adults in the middle belt of Ghana," PLoS ONE, vol. 7, no. 4, Article ID e36308, 2012.

[11] S. Owusu-Agyei, K. P. Asante, M. Adjuik et al., "Epidemiology of malaria in the forest-savanna transitional zone of Ghana," Malaria Journal, vol. 8, no. 1, article 220, 2009.

[12] K. P. Asante, S. Owusu-Agyei, M. Cairns et al., "Placental malaria and the risk of malaria in infants in a high malaria transmission area in Ghana: a prospective cohort study," Journal of Infectious Diseases, vol. 208, no. 9, pp. 1504-1513, 2013.

[13] F. Baiden, J. Webster, M. Tivura et al., "Accuracy of rapid tests for malaria and treatment outcomes for malaria and non-malaria cases among under-five children in rural ghana," PLOS ONE, vol. 7, no. 4, Article ID e34073, 2012.

[14] S. Zlotkin, S. Newton, A. M. Aimone et al., "Effect of iron fortification on malaria incidence in infants and young children in Ghana: a randomized trial," The Journal of the American Medical Association, vol. 310, no. 9, pp. 938-947, 2013.

[15] D. K. Dosoo, K. P. Asante, K. Kayan et al., "Biochemical and hematologic parameters for children in the middle belt of Ghana," The American Journal of Tropical Medicine and Hygiene, vol. 90, no. 4, pp. 767-773, 2014.

[16] B. M. Greenwood and J. R. M. Armstrong, "Comparison of two simple methods for determining malaria parasite density," Transactions of the Royal Society of Tropical Medicine and Hygiene, vol. 85, no. 2, pp. 186-188, 1991.

[17] P. Olliaro, A. Djimdé, C. Karema et al., "Standardised versus actual white cell counts in estimating thick film parasitaemia in African children under five," Tropical Medicine \& International Health, vol. 16, no. 5, pp. 551-554, 2011. 


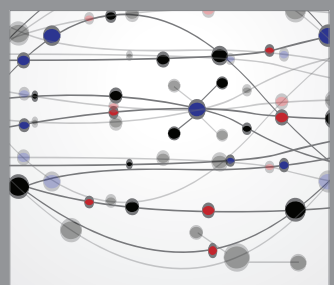

The Scientific World Journal
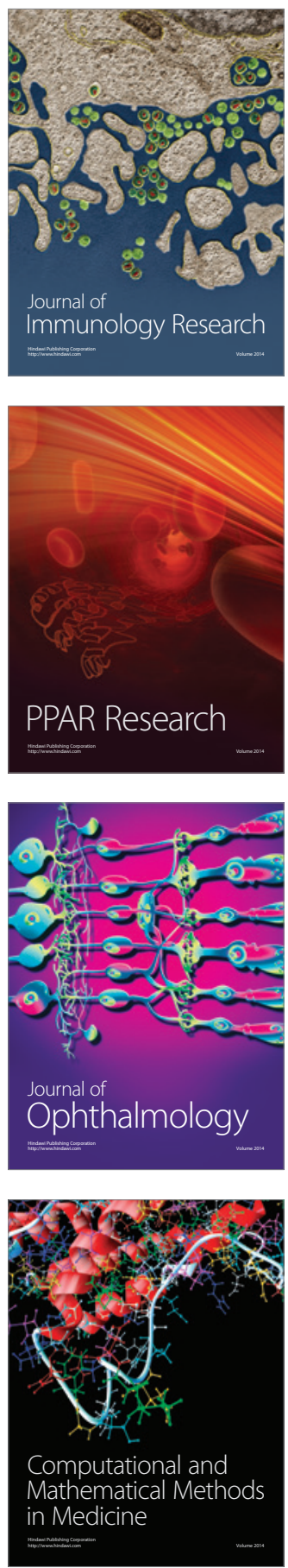

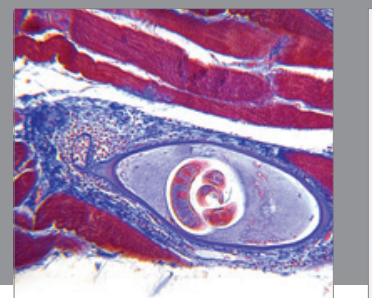

Gastroenterology

Research and Practice
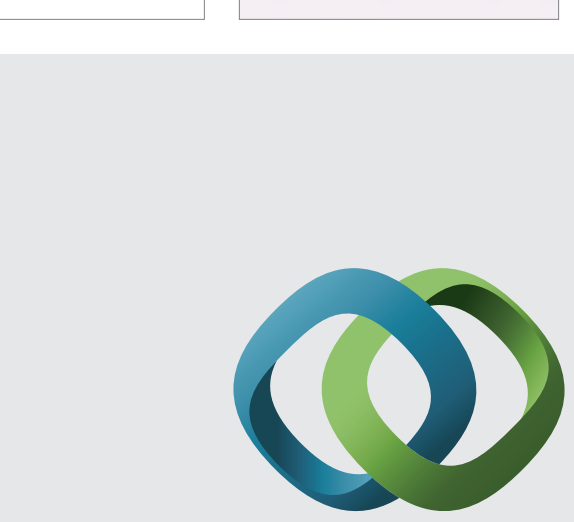

\section{Hindawi}

Submit your manuscripts at

http://www.hindawi.com
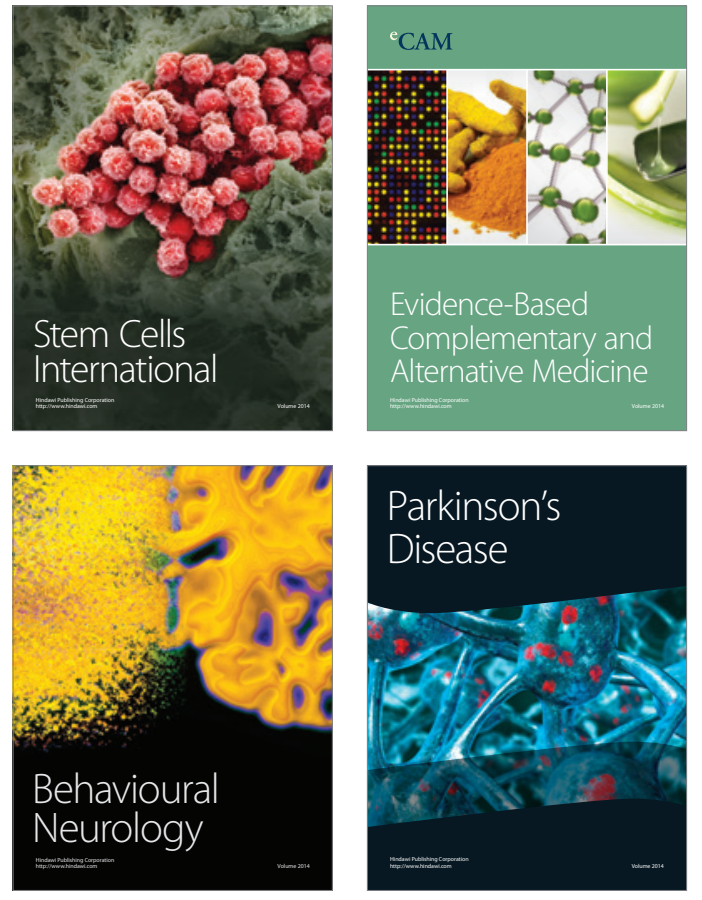
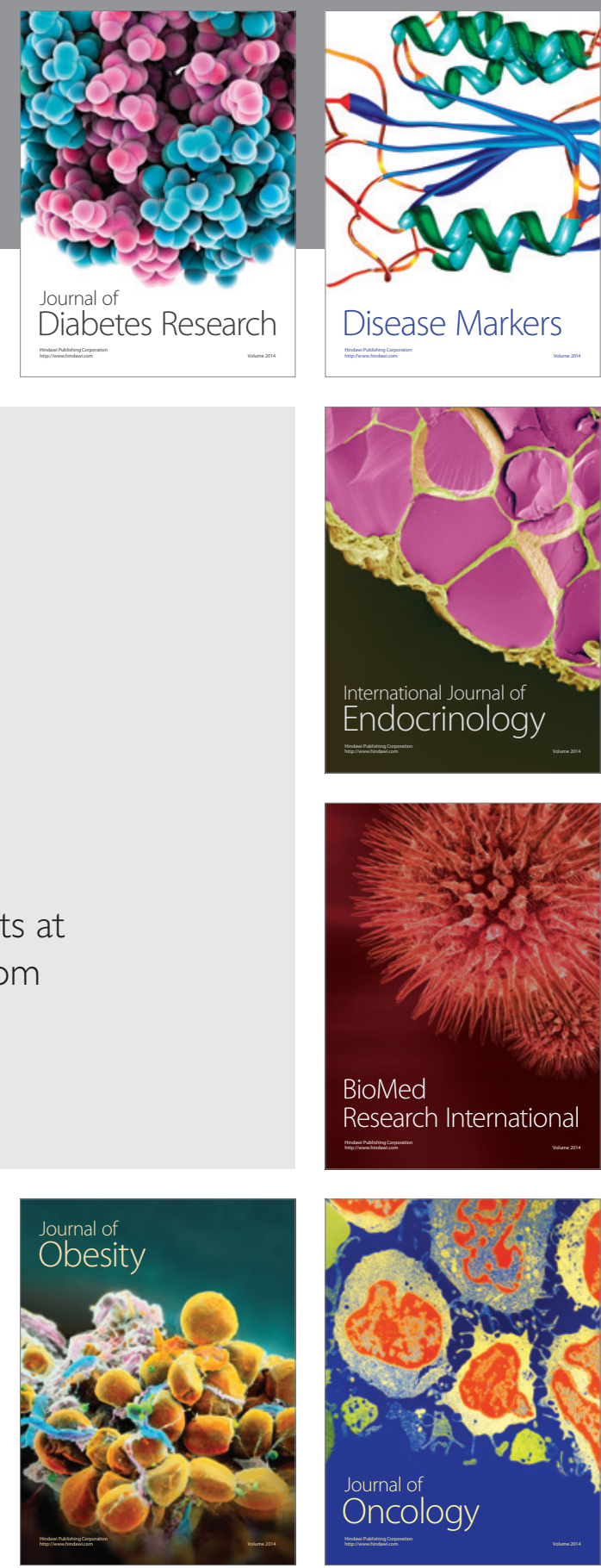

Disease Markers
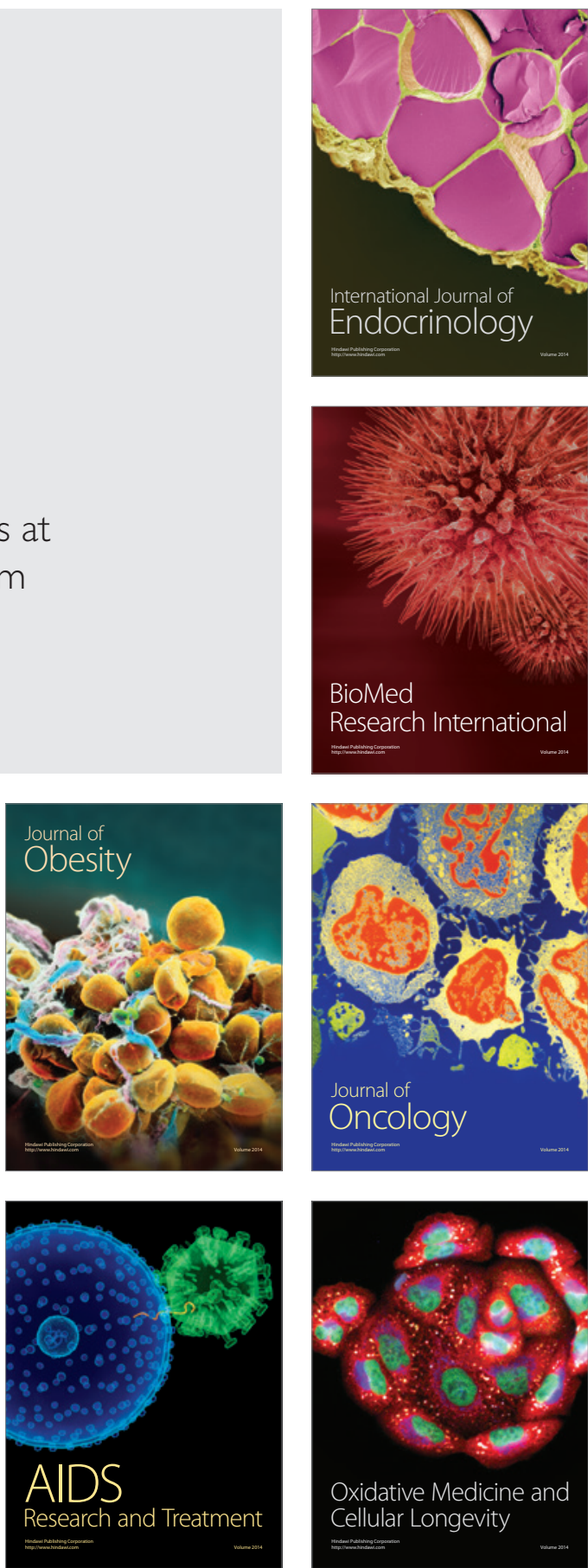